\title{
The flow induced by yawing motion in unidirectionally solidifying alloys
}

\author{
C.A. Chung, Falin Chen* \\ Institute of Applied Mechanics, National Taiwan University, Taipei 10764, Taiwan, ROC \\ Received 12 February 1999; accepted 1 June 1999 \\ Communicated by T. Nishinaga
}

\begin{abstract}
As the cooling tank is yawing, there occurs an induced flow in the binary solution cooled from below. The induced flow may stabilize the potentially unstable solution during directional solidification and suppress the formation of freckles in the cast alloys. We investigate this induced flow by an analytical approach. Results show that the induced flow in the melt region consists of three paralleling shear flows of different length scales. The one in the vicinity above the melt $/ \mathrm{mush}$ interface has the largest velocity gradient. The amplitude of the induced velocity in the melt is much larger than that in the dendritic mushy region, and the former is virtually inversely proportional to the yawing frequency while the latter is completely independent of the yawing frequency. The amplitudes of the induced velocities in both the melt and the mush are proportional to the yawing amplitude. The phase of induced flow in the melt lags by about $90^{\circ}$ behind the yawing motion while the induced flow in the mush is of the same phase with yawing. The sharp velocity gradient of the induced flow in the shallow layer above the melt/mush interface may play a crucial role in stabilizing the potentially very unstable fluid in the solute boundary layer above the interface. This suggests that the yawing motion may be an efficient scheme for the prohibition of the occurrence of the plume convection and for the suppression of the formation of freckles as well. (C) 1999 Elsevier Science B.V. All rights reserved.
\end{abstract}

\section{Introduction}

It has been shown from previous investigations, for example Refs. [1-4] and references therein, that in the directional solidification of binary alloys there exist various kinds of convective flows which will to some extent influence the final mechanical properties of the casting. According to the categorization of the convective flows defined by Chen [5], the various kinds are salt-finger convection, plume convection, bulk fluid convection, and double-

\footnotetext{
* Corresponding author. Fax: + 886-2-3639290.
}

diffusive layer convection, and so on. Regarding the salt-finger convection, there are two instability modes competing to dominate for the onset of convection $[2,4]$. One is the boundary-layer mode, which is a convective flow confined to the shallow solute boundary layer above the melt/mush interface. The other is the mushy-layer mode, which is a convective flow of larger length scale, circulating mostly in the mushy zone which will under some circumstances drive the fluid above the melt/mush interface through the viscous force to form another convection cell [4]. Either one of these two modes will dominate the initial phase of convection in the system and develop further into a nonlinear 
convective flow. This nonlinear flow imposes a strong perturbation to the system and triggers subcritically the formation of the plume convection [6-8], a buoyant flow passing through vertical channels in the mush. It is this plume convection which is responsible for the formation of freckles in the casting. Since the appearance of the freckles deteriorates dramatically the mechanical properties of the casting, prevention of the formation of a plume has therefore become one of the major issues to which most of the research efforts in directional solidification have been devoted. The major goal of these research efforts has been to find efficient schemes to inhibit the plume convection.

To achieve this goal, Sample and Hellawell [9] proposed rotating the cooling tank with respect to an axis with an inclined angle varying from 0 to $30^{\circ}$. From a series of experiments they found that when the inclined angle lay within $20-30^{\circ}$ and the tank rotated with spin and/or precession with respect to the inclined axis, the plume convection could be successfully inhibited. They attributed this success to the appearance of the paralleling shear flow above the melt/mush interface induced by the inclined rotation. We note that both Neilson and Incropera [10], and $\mathrm{Lu}$ and Chen [11] have shown that rotation might not be a good way to suppress the plume convection when the rotation axis is vertical because under this case there is no induced flow due to rotation. Another way to inhibit the formation of plume convection was proposed by Huppert and Hallworth [12], who added small quantities of copper sulfate into the solution of $27 \mathrm{wt} \%$ ammonium chloride solution in water. They showed that as the quantity of the contamination increased, the time taken for the plumes to appear in the system increased. When the contamination increased to $0.5 \mathrm{wt} \%$ or above, no plume was observed. The disappearance of the plume is due to the fact that the increase of the contamination leads to an increase of the solid fraction of the dendritic mushy layer and then to the inhibition of the plume convection.

In the present paper, we propose another scheme with which one can also successfully prohibit the occurance of the plume convection. We suggest that the tank be yawing in the vertical plane (or with respect to the $\boldsymbol{x}$-axis) such that a strong shear flow above the melt/mush interface is induced. This is expected to have similar effect on the plume convetion as that due to the inclined spin and/or precession [9]. We calculate this induced flow by an analytical approach. From the analytical solution we are able to identify the charecteristics of the induced flow and the variation of the shear flow with respect to various yawing situations. On the bases of these analyses an efficient and practical scheme for directional solidification may thus be created.

We describe in Section 2 the problem investigated and the corresponding mathematical formulations. We then in Section 3 solve the governing partial differential equations and the associated boundary conditions analytically. In this section the assumptions required to simplify the mathematical model are also discussed. The analytical solutions are presented for two different cases: one is for the case in which the yawing angle is small, the other is for the yawing angle arbitrarily large. It turns out that the small yawing-angle case, being in a more simplified mathematical form, can lead to a result which can still account for most of the cases considered. Finally the conclusions are given in Section 4.

\section{Problem description and formulations}

Consider the system as shown in Fig. 1, a binary solution of concentration $C_{\infty}$ and temperature $T_{\infty}$ being unidirectionally solidified from below. A dendritic mush, extending from $z=0$ to $z=$ $h(x, y, t)$, forms between the eutectic solid below and the semi-infinite bulk fluid above. The height of the mush $h$ is to be determined as part of the solution. Assume that the solid/mush interface moves in the direction of the thermal gradient with a constant velocity $V$. The tank yaws in the vertical plane (or with respect to the $\boldsymbol{x}$-axis) in a simply periodical way. The yawing angle can be described as

$\phi=\Phi \sin (\omega t)$,

where $\Phi$ is the amplitude of yawing and $\omega$ is the angular frequency. The coordinate is fixed on the 


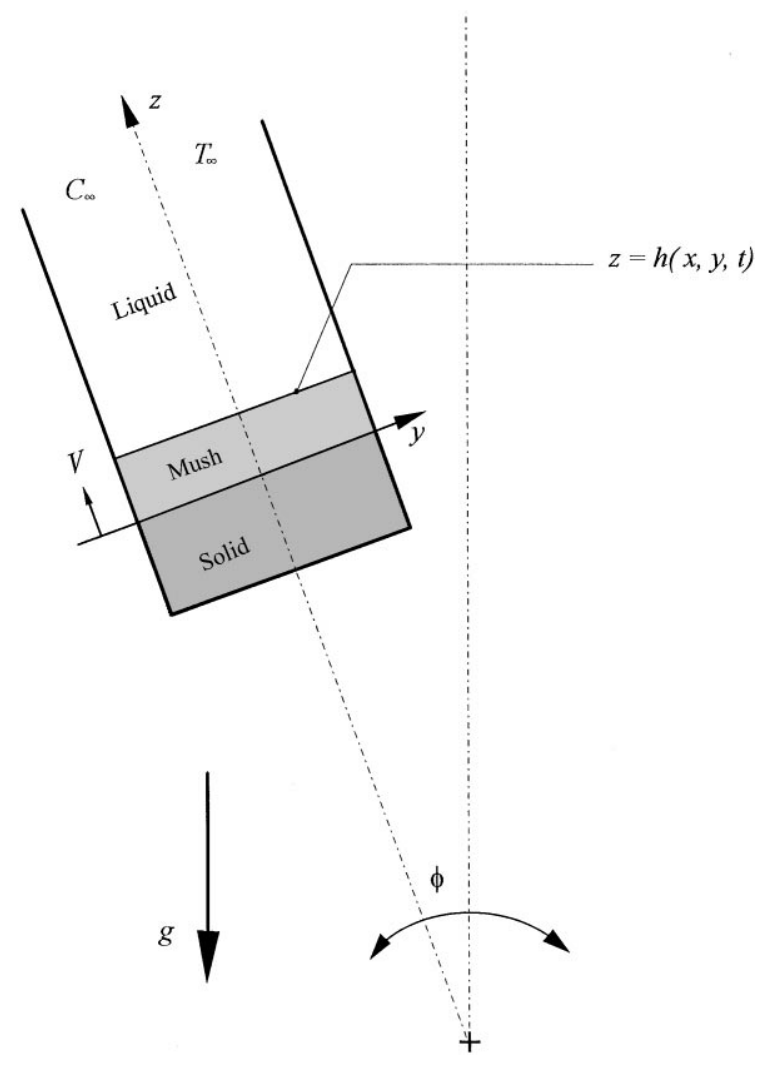

Fig. 1. The system considered. The cooling tank is yawing in the $y-z$ plane, where $\phi$ accounts for the yawing angle and $g$ accounts for the gravity acting vertically downwards. In the cooling tank, there exits a dendritic mushy layer overlying the eutectic solid and underlying the bulk melt. The coordinate system is fixed on the mush/solid interface, which moves with a constant speed $V$ along the $z$-axis. The position of the melt/mush interface $h(x, y, t)$ is to be solved.

mush/solid interface, translating with $V$ and yawing with $\dot{\phi}$ (Fig. 1). The $\dot{\phi}$ is the yawing angular velocity, can be obtained by differentiating Eq. (2.1) with time, yielding

$\dot{\phi}=\Phi \omega \cos (\omega t) \boldsymbol{e}_{x}$,

where $\boldsymbol{e}_{x}$, together with $\boldsymbol{e}_{y}$ and $\boldsymbol{e}_{z}$ are the unit vectors of the Cartesian coordinate, which is denoted by $\boldsymbol{y}-\boldsymbol{z}$ in Fig. 1 . Note that the $\boldsymbol{x}$-axis points out of the page and is thus not shown in the figure.

The equations governing the fluid motion in the tank can be divided into two sets, one in the fluid layer (or the bulk melt) and one in the mushy layer. Under the coordinate system described above, the governing equations in the fluid layer $h<z<\infty$ are

$\nabla \cdot \boldsymbol{u}=0$,

$$
\begin{aligned}
{\left[\frac{\partial}{\partial t}-V \frac{\partial}{\partial z}+\boldsymbol{u} \cdot \nabla\right] \boldsymbol{u}=} & -\nabla P+v \nabla^{2} \boldsymbol{u} \\
& +\left(\frac{\rho}{\rho_{0}}-1\right) \boldsymbol{g}+2 \boldsymbol{u} \times \dot{\boldsymbol{\phi}},
\end{aligned}
$$

$$
\begin{aligned}
& \left(\frac{\partial}{\partial t}-V \frac{\partial}{\partial z}+\boldsymbol{u} \cdot \nabla\right) T=\kappa \nabla^{2} T, \\
& \left(\frac{\partial}{\partial t}-V \frac{\partial}{\partial z}+\boldsymbol{u} \cdot \nabla\right) C=D_{f} \nabla^{2} C .
\end{aligned}
$$

In the above equations, $\boldsymbol{u}$ accounts for the velocity vector $(u, v, w)$ with respect to the cooling tank, $P=\left(p / \rho_{0}\right)-\boldsymbol{g} \cdot \boldsymbol{r}, \quad p$ is the static pressure, $\rho_{0}$ is the reference density, and $r$ is the position vector with respect to the transformed coordinate. Besides, $T$ is the temperature, $C$ the concentration, $\kappa$ the thermal diffusivity, $D_{f}$ the solute diffusivity, $v$ the kinematic viscosity, and $\boldsymbol{g}=$ $-g(0, \sin \phi, \cos \phi)$ the vector of gravitational acceleration, changing with the yawing angle, and $g$ is the gravitational constant. Since the Boussinesq approximation is applied, the density of the fluid is a constant except in the gravity term in which the following relation holds:

$\rho=\rho_{0}\left(1-\alpha^{*}\left(T-T_{\mathrm{L}}\left(C_{\infty}\right)\right)+\beta^{*}\left(C-C_{\infty}\right)\right)$,

where $\alpha^{*}$ and $\beta^{*}$ are, respectively, the thermal and solute expansion coefficients, and $T_{\mathrm{L}}\left(C_{\infty}\right)$ is the liquidus temperature corresponding to $C_{\infty}$.

In the mushy layer $0<z<h$, the governing equations are

$\nabla \cdot \boldsymbol{u}=0$,

$\frac{v}{\Pi(\chi)} \boldsymbol{u}=-\nabla P+\left(\frac{\rho}{\rho_{0}}-1\right) \boldsymbol{g}+\frac{2}{\chi} \boldsymbol{u} \times \dot{\boldsymbol{\phi}}$,

$\left(\frac{\partial}{\partial t}-V \frac{\partial}{\partial z}+\boldsymbol{u} \cdot \nabla\right) T=\kappa \nabla^{2} T-\frac{\mathscr{L}}{c}\left(\frac{\partial}{\partial t}-V \frac{\partial}{\partial z}\right) \chi$, 


$$
\begin{aligned}
& \left(\frac{\partial}{\partial t}-V \frac{\partial}{\partial z}\right)\left[\chi C+(1-\chi) C_{\mathrm{s}}\right]+\boldsymbol{u} \cdot \nabla C \\
& =\nabla \cdot\left(\chi D_{f} \nabla C\right) .
\end{aligned}
$$

In the above equations, $\Pi(\chi)$ is the permeability function, $\chi$ the porosity, $\mathscr{L}$ the latent heat per unit volume, $c$ the specific heat per unit volume, and $C_{\mathrm{s}}$ the concentration of the solid. The specific heat coefficients, the thermal conductivities and the solute diffusivities of both the liquid and solid phases are assumed equal [2]. In Eqs. (2.3b) and (2.5b) we have neglected the centrifugal and tangential acceleration since their relative significance to the gravity is small on the assumption that the yaw frequency is low. Since thermodynamic equilibrium holds in the mush [4], the liquidus relation

$T-T_{\mathrm{L}}\left(C_{\infty}\right)=\Gamma\left(C-C_{\infty}\right)$,

is applied, where $\Gamma$ is the slope of the liquidus. As a result, the density in the mush can be represented by

$\rho=\rho_{0}\left(1+\bar{\beta}\left(C-C_{\infty}\right)\right)$,

where $\bar{\beta}=\beta^{*}-\Gamma \alpha^{*}$.

Regarding the boundary conditions at infinite far field, both the temperature and the concentration are assumed to remain unchanged and the fluid remains quiescent with respect to the tank, corresponding to the so-called rigid-body rotation (in fact only yawing) condition. Besides, the height of the tank is assumed large enough that the influence on the induced-flow due to the possible deformation of the free surface at far field can be neglected. Accordingly, at $z \rightarrow \infty$ we have

$$
\begin{aligned}
T & \rightarrow T_{\infty}, \\
C & \rightarrow C_{\infty}, \\
\boldsymbol{u} & \rightarrow \mathbf{0} .
\end{aligned}
$$

At the melt/mush interface $z=h(x, y, t)$ the boundary conditions are

$$
T-T_{\mathrm{L}}\left(C_{\infty}\right)=\Gamma\left(C-C_{\infty}\right),
$$

$\frac{\partial T}{\partial n}=\Gamma \frac{\partial C}{\partial n}$

$$
\begin{aligned}
& {[\boldsymbol{u} \cdot \boldsymbol{n}]=0,} \\
& {[T]=0,} \\
& {\left[\frac{\partial T}{\partial n}\right]=0,} \\
& \chi=1, \\
& {\left[\sigma_{n}\right]=0,} \\
& \left.\frac{\partial \boldsymbol{u}_{2}}{\partial n}\right|_{h^{+}}=\frac{\Lambda}{\Pi(1)}\left(\left.\boldsymbol{u}_{2}\right|_{h^{+}}-\left.\boldsymbol{u}_{2}\right|_{h^{-}}\right),
\end{aligned}
$$

where [] accounts for the jump of the enclosed quantity across the interface, $\boldsymbol{n}$ is the normal vector to the interface, and $\sigma_{n}$ is the normal stress. Eq. (2.9a) represents the concentration continuity. Eq. $(2.9 \mathrm{~b})$ is the marginal equilibrium condition proposed by Worster [13]. Eqs. (2.9c), (2.9d) and (2.9e) account respectively for the mass, temperature, and the heat flux continuities. Eq. (2.9f) states that the porosity is fixed to be unity. Eq. $(2.9 \mathrm{~g})$ describes the normal stress continuity. Eq. (2.9h) is an empirical condition proposed by Beavers and Joseph [14], describing the relation between the planar velocities in the fluid and mushy layers, in which $\boldsymbol{u}_{2}$ is the planar velocity vector $(u, v)$ and $\Lambda$ is an empirical constant determined by experiment. With regards to the boundary conditions at mush/solid interface, the eutectic concentration (or temperature) and the impermeable boundary conditions are prescribed; namely, at $z=0$ we have

$$
\begin{gathered}
T=T_{\mathrm{E}}, \\
\boldsymbol{u} \cdot \boldsymbol{n}=0,
\end{gathered}
$$

where $T_{\mathrm{E}}$ is the eutectic temperature of the solid.

We nondimensionalize the governing equations as well as the boundary conditions with the following scales: $V$ for velocity, $H=\kappa / V$ for length, $\kappa / V^{2}$ for time, and $\bar{\beta} \Delta C \rho_{0} g \kappa / V$ for pressure. In which, $\Delta C=C_{\infty}-C_{\mathrm{E}}$ and $C_{\mathrm{E}}$ is the eutectic concentration. The dimensionless temperature and concentration are respectively

$$
\begin{aligned}
& \theta=\frac{T-T_{\mathrm{L}}\left(C_{\infty}\right)}{\Delta T}, \\
& \Theta=\frac{C-C_{\infty}}{\Delta C},
\end{aligned}
$$


where $\Delta T=\Gamma \Delta C=T_{\mathrm{L}}\left(C_{\infty}\right)-T_{\mathrm{E}}$. For convenience we adopt the same notation for the variables in the previous equations, such as the velocity, pressure, permeability, time, space, and so on, for the dimensionless counterparts in the following equations. As a result, in the fluid layer, the dimensionless continuity equation, momentum equation, heat balance and solute balance equations are respectively

$\nabla \cdot \boldsymbol{u}=0$,

$\frac{1}{\sigma}\left[\frac{\partial}{\partial t}-\frac{\partial}{\partial z}+\boldsymbol{u} \cdot \nabla\right] u=\nabla^{2} u-\mathscr{H} R_{\mathrm{m}} \frac{\partial p}{\partial x}$,

$\frac{1}{\sigma}\left[\frac{\partial}{\partial t}-\frac{\partial}{\partial z}+\boldsymbol{u} \cdot \nabla\right] v=\nabla^{2} v-\mathscr{H} \boldsymbol{R}_{\mathrm{m}} \frac{\partial p}{\partial y}$

$$
\begin{aligned}
& +\left(R_{\mathrm{t}} \theta-R_{\mathrm{c}} \Theta\right) \sin (\phi) \\
& +T_{\mathrm{a}}^{1 / 2} w \cos (\Omega t),
\end{aligned}
$$

$\frac{1}{\sigma}\left[\frac{\partial}{\partial t}-\frac{\partial}{\partial z}+\boldsymbol{u} \cdot \nabla\right] w=\nabla^{2} w-\mathscr{H} R_{\mathrm{m}} \frac{\partial p}{\partial z}$

$$
+\left(R_{\mathrm{t}} \theta-R_{\mathrm{c}} \Theta\right) \cos (\phi)
$$$$
-T_{\mathrm{a}}^{1 / 2} v \cos (\Omega t)
$$

$\left(\frac{\partial}{\partial t}-\frac{\partial}{\partial z}+\boldsymbol{u} \cdot \nabla\right) \theta=\nabla^{2} \theta$

$\left(\frac{\partial}{\partial t}-\frac{\partial}{\partial z}+\boldsymbol{u} \cdot \nabla\right) \Theta=\varepsilon \nabla^{2} \Theta$.

In the mushy layer, the continuity equation, Darcy equation, heat and solute balance equations are respectively

$\nabla \cdot \boldsymbol{u}=0$,

$\frac{u}{\Pi(\chi)}=-R_{\mathrm{m}} \frac{\partial p}{\partial x}$,

$\frac{v}{\Pi(\chi)}=-R_{\mathrm{m}} \frac{\partial p}{\partial y}-R_{\mathrm{m}} \theta \sin (\phi)+T_{\mathrm{m}}^{1 / 2} \frac{w}{\chi} \cos (\Omega t)$,

$\frac{w}{\Pi(\chi)}=-R_{\mathrm{m}} \frac{\partial p}{\partial z}-R_{\mathrm{m}} \theta \cos (\phi)-T_{\mathrm{m}}^{1 / 2} \frac{v}{\chi} \cos (\Omega t)$,

$$
\begin{aligned}
& \left(\frac{\partial}{\partial t}-\frac{\partial}{\partial z}+\boldsymbol{u} \cdot \nabla\right) \theta=\nabla^{2} \theta-\mathscr{F}\left(\frac{\partial}{\partial t}-\frac{\partial}{\partial z}\right) \chi, \\
& \chi\left(\frac{\partial}{\partial t}-\frac{\partial}{\partial z}\right) \theta+\boldsymbol{u} \cdot \nabla \theta=-(\theta-\mathscr{C})\left(\frac{\partial}{\partial t}-\frac{\partial}{\partial z}\right) \chi .
\end{aligned}
$$

In the above equations, $\sigma \equiv v / \kappa$ is the Prandtl number, $\mathscr{H} \equiv H^{2} / \Pi_{0}$ is the inverse Darcy number, $\Pi_{0}$ is the reference permeability of the mush, $R_{\mathrm{t}}$ and $R_{\mathrm{c}}$ are, respectively, the thermal and solute Rayleigh numbers of the fluid layer, and $R_{\mathrm{m}}$ is the Rayleigh number of the mushy layer. They are defined as

$$
\begin{aligned}
& R_{\mathrm{t}} \equiv \frac{g \alpha^{*} \Delta T H^{3}}{\kappa v}, \\
& R_{\mathrm{c}} \equiv \frac{g \beta^{*} \Delta C H^{3}}{\kappa v}, \\
& R_{\mathrm{m}} \equiv \frac{g \bar{\beta} \Delta C \Pi_{0} H}{\kappa v} .
\end{aligned}
$$

It is noted that $R_{\mathrm{t}}, R_{\mathrm{c}}$ and $R_{\mathrm{m}}$ satisfy the relations

$R_{\mathrm{t}}=\mathscr{A} \mathscr{H} R_{\mathrm{m}}$

$R_{\mathrm{c}}=(1+\mathscr{A}) \mathscr{H} R_{\mathrm{m}}$

where $\mathscr{A} \equiv \Gamma \alpha^{*} / \bar{\beta}$ is the buoyancy ratio. In addition, $\varepsilon \equiv D_{f} / \kappa$ is the inverse Lewis number, $\mathscr{F} \equiv$ $\mathscr{L} /(c \Delta T)$ is the Stephan number, $\mathscr{C} \equiv\left(C_{\mathrm{s}}-C_{\infty}\right) /$ $\Delta C$ is the concentration ratio. Since the $\varepsilon$ of the mush is very small, we neglect the mass diffusion in the mush. Meanwhile, because of the thermodynamic equilibrium condition (Eq. (2.6)), the nondimensional liquidus relation in the mush can be written as $\theta=\Theta$, which has been introduced into Eqs. (2.13c), (2.13d) and (2.13f). The dimensionless yawing angular frequency $\Omega \equiv \omega \kappa / V^{2}$ is used to rewrite the yawing angle $\phi$ as

$\phi=\Phi \sin (\Omega t)$

The dimensionless parameters $T_{\mathrm{a}}$ and $T_{\mathrm{m}}$ account, respectively, for Taylor numbers in the bulk 
melt and mushy layer, being defined as

$$
\begin{aligned}
& T_{\mathrm{a}} \equiv\left(\frac{2 H^{2} \Phi \omega}{v}\right)^{2}, \\
& T_{\mathrm{m}} \equiv\left(\frac{2 \Pi_{0} \Phi \omega}{v}\right)^{2}=\frac{T_{\mathrm{a}}}{\mathscr{H}^{2}} .
\end{aligned}
$$

The boundary conditions at $z \rightarrow \infty$ are

$$
\begin{gathered}
\theta \rightarrow \theta_{\infty}, \\
\Theta \rightarrow 0, \\
\boldsymbol{u} \rightarrow \mathbf{0},
\end{gathered}
$$

at the melt/mush interface $z=h(x, y, t)$ are

$\theta=\Theta$

$\frac{\partial \theta}{\partial n}=\frac{\partial \Theta}{\partial n}$

$[\boldsymbol{u} \cdot \boldsymbol{n}]=0$,

$[\theta]=0$,

$\left[\frac{\partial \theta}{\partial n}\right]=0$

$\chi=1$,

$\left[\sigma_{n}\right]=0$,

$\left.\frac{\partial \boldsymbol{u}_{2}}{\partial n}\right|_{h^{+}}=\Lambda \sqrt{\frac{\mathscr{H}}{\Pi(1)}}\left(\left.\boldsymbol{u}_{2}\right|_{h^{+}}-\left.\boldsymbol{u}_{2}\right|_{h^{-}}\right)$,

and at $z=0$ are

$\theta=-1$

$\boldsymbol{u} \cdot \boldsymbol{n}=0$.

We note from Eqs. (2.12a), (2.12b), (2.12c), (2.12d), (2.12e), (2.12f), (2.13a), (2.13b), (2.13c), (2.13d), (2.13e) and (2.13f) that the fluid of the tank can be driven under the action of the pressure gradient, the gravity, and the Coriolis force. Note also that the pressure gradient is hydrostatically balanced with the weight of the fluid, resulting in a quiescent state in the system before the induced flow occurs. The pressure gradient is therefore equivalent to "the buoyancy" (which is of more physical sense intuitively), and will be referred as the buoyancy hereafter. The terms accounting for the Coriolis force are those containing $\cos (\Omega t)$, being on the right-hand side of Eqs. (2.12c), (2.12d), (2.13c) and (2.13d). As will be shown in the next section, the Coriolis force is of no influence on the induced flow. Only the buoyancy and the gravity affect the flow structure, driving the fluid to move parallel along the melt/mush interface.

In the following section, based on Eqs. (2.12a), (2.12b), (2.12c), (2.12d), (2.12e), (2.12f), (2.13a), (2.13b), (2.13c), (2.13d), (2.13e) and (2.13f) and the associated boundary conditions Eqs. (2.18a), (2.18b), (2.18c), (2.19a), (2.19b), (2.19c), (2.19d), (2.19e), (2.19f), (2.19g), (2.19h), (2.20a) and (2.20b), the flow induced by the yawing motion will be solved analytically and some typical examples will be shown.

\section{Yawing-induced flow}

Before the onset of the convection due to solidification, there is a flow induced by the yawing motion of the tank. The induced flow is a result of the interaction between the gravity and the buoyancy. The direction of the buoyancy changes with time as the tank is yawing while the direction of the gravity remains pointing vertically downwards. The combination of these two forces therefore changes with time periodically, and so does the induced flow.

\subsection{Assumptions}

To investigate the induced flow, we need to solve Eqs. (2.12a), (2.12b), (2.12c), (2.12d), (2.12e), (2.12f), (2.13a), (2.13b), (2.13c), (2.13d), (2.13e) and (2.13f) together with the associated boundary conditions Eqs. (2.18a), (2.18b), (2.18c), (2.19a), (2.19b), (2.19c), (2.19d), (2.19e), (2.19f), (2.19g), (2.19h), (2.20a) and (2.20b) which are nevertheless too complicate to be solved without assumptions. To simplify the problem, we first assume that the horizontal dimension of the $\operatorname{tank} L$ is much larger than the characteristic length of the solidification $H$, which is about $1 \mathrm{~cm}$ in experiments for most binary solutions [1,9]. 
Namely, we have

$\frac{L}{H} \gg 1$.

The second assumption to be made is that the small-scale convection is absent from the system when the induced flow is driven. This assumption is usually valid because the induced flow occurs before the onset of small-scale convection in the directionally solidifying binary alloy [9]. Consequently, one finds from the scale analysis of the continuity equation that

$\frac{U}{L} \approx \frac{W}{H}$,

where $U$ and $W$ account, respectively, for the characteristic velocities in the $\boldsymbol{x}-\boldsymbol{y}$ plane and the $\boldsymbol{z}$ direction. Eqs. (3.1) and (3.2) imply that $W \ll U$, saying that the velocity in the $z$-direction is negligible compared with the velocity along the $\boldsymbol{x}-\boldsymbol{y}$ plane. Furthermore, to satisfy Eq. (3.1), one can also derive from the scale analysis that the diffusivities in both the $\boldsymbol{x}$ - and $\boldsymbol{y}$-directions are negligible when compared to that in the $z$-direction, i.e.

$\frac{\partial^{2}}{\partial x^{2}} \ll \frac{\partial^{2}}{\partial z^{2}}, \frac{\partial^{2}}{\partial y^{2}} \ll \frac{\partial^{2}}{\partial z^{2}}$.

We also note that as the tank is yawing, the direction of the buoyancy changes periodically by a frequency equal to the yawing angular frequency $\omega$. As a result, there exists a characteristic time scale of the system as $\omega^{-1}$. If we consider that the Strouhal number of yawing is large, we have

$\frac{\omega L}{U} \gg 1$

By dividing both sides of Eq. (3.4) by $H$ and by employing the definition of the dimensionless yawing angular frequency, Eq. (3.4) can be rewritten as

$\frac{L}{H} \gg \frac{U}{\omega H}=\frac{U}{\Omega V}$.

Based on above results of scale analyses, both the temperature and the concentration fields and the flow induced by the yawing motion can be solved analytically, which is shown in the following section.

\subsection{Temperature and concentration fields}

Due to the absence of the small-scale fluid motion, the nonlinear advection term can also be neglected when compared with the unsteady term. Consequently, the temperature and concentration can be decoupled from the velocity. In other words, both the temperature and the concentration fields are not influenced by the presence of the flow induced by the yawing motion. They therefore shall be the same as those shown in Worster [2], in which the system is motionless before the onset of convection.

For the convenience of subsequent discussions, both the temperature and the concentration distributions are shown in the following. In the fluid layer, their distribution functions can be explicitly expressed as

$\theta=\theta_{\infty}+\left(\theta_{\mathrm{i}}-\theta_{\infty}\right) \exp [-(z-h)]$,

$\Theta=\theta_{\mathrm{i}} \exp \left[-\left(\frac{z-h}{\varepsilon}\right)\right]$,

where $\theta_{\mathrm{i}}$ is the temperature (or concentration) at the melt/mush interface

$\theta_{\mathrm{i}}=\left(\frac{-\varepsilon}{1-\varepsilon}\right) \theta_{\infty}$,

and $h$ is the mushy-layer height, which is now a constant. In the mushy layer the temperature distribution $\theta$ can only be expressed by the implicit function

$z=\frac{\alpha-\mathscr{C}}{\alpha-\beta} \ln \left(\frac{\alpha+1}{\alpha-\theta}\right)+\frac{\mathscr{C}-\beta}{\alpha-\beta} \ln \left(\frac{\beta+1}{\beta-\theta}\right)$

and the porosity is a function of $\theta$

$\chi=\frac{\mathscr{C}-\theta_{\mathrm{i}}}{\mathscr{C}-\theta}$

where

$\alpha=A+B$,

$\beta=A-B$, 
$A=\frac{1}{2}\left(\mathscr{C}+\theta_{\infty}+\mathscr{F}\right)$,

$B=\sqrt{A^{2}-\mathscr{C} \theta_{\infty}-\mathscr{F} \theta_{\mathrm{i}}}$.

Note that the mushy-layer height $h$ can be obtained by substituting $\theta=\theta_{\mathrm{i}}$ into Eq. (3.8). An example of the temperature and concentration distributions and the associated density distribution corresponding to the $26 \%$ ammonium chloride solution is shown in Fig. 2. It is seen that in the mushy layer both the temperature and the concentration increase exponentially with height. In the fluid layer, nevertheless, the concentration remains essentially the same while the temperature also increases monotonically upwards. Regarding the density distribution, as shown in Fig. 2b, the density distribution due to temperature decreases exponentially upwards in both the fluid and the mushy layers while that due to concentration increases with height in the mushy layer and remains virtually unchanged in the fluid layer. The overall density distribution shows that in the mushy layer the density is an increasing function with height while in the fluid layer it turns into a decreasing function with height. In the viewpoint of hydrodynamic stability, the fluid in the mushy layer is potentially unstable while in the fluid layer is quite stable. There is nonetheless an exception that in the shallow solute boundary layer above the melt/mush interface whose thickness is of $\mathrm{O}(\varepsilon)$, the overall density increases with height. As a result, although the density in both the mushy layer and the solute boundary layer increases with height, the fluid in the solute boundary layer is normally more unstable. This is because, in the mush the dendritic solid imposes large resistance to the fluid such that the fluid cannot move easily and freely while that in the solute boundary layer can move around relatively more easily. Consequently, the onset of convection occurs first in the solute boundary layer [4].

We nevertheless note also that, before the onset of convection occurs, there is a large parameter range in terms of Rayleigh numbers in which the fluid remains stable with respect to the solidification effect. In this parameter range, the interaction between the buoyancy and the gravity can become active when the tank is yawing. As the tank moves into an attitude in which the axis of the tank is at an inclined angle with respect to the vertical axis, the gravity and the buoyancy can no longer be balanced with each other. Their combination is of a net component along the melt/mush interface, which eventually drives the fluid to move along the interface. We shall show this induced flow in the following section.
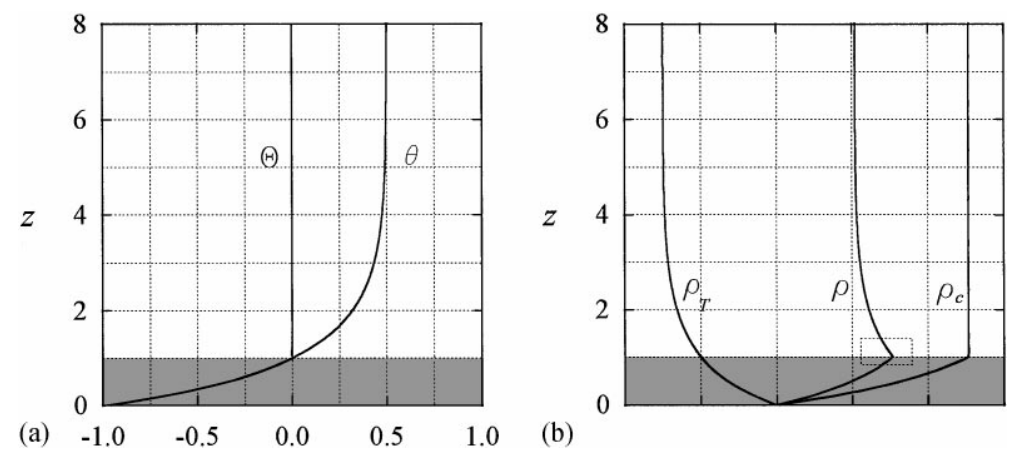

(b)

Fig. 2. In (a) the temperature distribution $\theta$ and the concentration distribution $\Theta$ in both the fluid and the mushy (shadowed region) layers are shown. In the mushy layer, since the thermodynamic equilibrium condition holds, we have $\theta=\Theta$. The temperature increases monotonically upwards in both the fluid and mushy layers. The concentration in the mush is also an increasing function with height, while in the melt it remains virtually constant. In (b), the density distribution due to temperature $\rho_{\mathrm{T}}$ and that due to concentration $\rho_{\mathrm{C}}$ and the total density distribution $\rho$ are shown. It is seen that $\rho_{\mathrm{T}}$ decreases invariably with height, while $\rho_{\mathrm{C}}$ increases monotonically with height in the mush but remains virtually unchanged in the melt. As a result, the overall density $\rho$ increases upwards in the mush but decreases in the melt. Please note that there exists a shallow solute boundary layer above the melt/mush interface, in which $\rho$ also increases upwards, as indicated by the small rectangular domain enclosed by the dashed lines. 


\subsection{Flow induced by yawing}

Eqs. (2.12b), (2.12c), (2.12d), (2.13b), (2.13c) and (2.13d) are to be solved to obtain the induced flow. We first apply the far-field rigid-body rotation (or yawing) condition, Eq. (2.18c) on Eqs. (2.12b), (2.12c) and (2.12d). Since at far field $u=v=w=0$, we find that the pressure distribution can be expressed in the following form:

$p=\bar{p}(z, t)+\mathscr{A} \theta_{\infty}[y \sin (\phi)+z \cos (\phi)]$,

in which the terms containing $A \theta_{\infty}$ account for the buoyant component along the melt/mush interface. We substitute Eqs. (3.6a), (3.6b), (3.7), (3.8) and (3.11) into Eqs. (2.12b), (2.12c), (2.12d), (2.13b), $(2.13 \mathrm{c})$ and $(2.13 \mathrm{~d})$, yielding that the velocity and the pressure satisfy the following simplified equations. In the fluid layer the equations are

$\left[\frac{1}{\sigma}\left(\frac{\partial}{\partial t}-\frac{\partial}{\partial z}\right)-\frac{\partial^{2}}{\partial z^{2}}\right] u=0$,

$\left[\frac{1}{\sigma}\left(\frac{\partial}{\partial t}-\frac{\partial}{\partial z}\right)-\frac{\partial^{2}}{\partial z^{2}}\right] v=\left[R_{\mathrm{t}}\left(\theta-\theta_{\infty}\right)-R_{\mathrm{c}} \Theta\right] \sin (\phi)$,

$w=0$,

$$
\begin{aligned}
\mathscr{H} R_{\mathrm{m}} \frac{\partial \bar{p}}{\partial z}=[ & \left.R_{\mathrm{t}}\left(\theta-\theta_{\infty}\right)-R_{\mathrm{c}} \Theta\right] \cos (\phi) \\
& -T_{\mathrm{a}}^{1 / 2} v \cos (\Omega t)
\end{aligned}
$$

and in the mushy layer the equations are

$u=0$,

$\frac{v}{\Pi}=-R_{\mathrm{m}}\left(\mathscr{A} \theta_{\infty}+\theta\right) \sin (\phi)$,

$w=0$,

$R_{\mathrm{m}} \frac{\partial \bar{p}}{\partial z}=-R_{\mathrm{m}}\left(\mathscr{A} \theta_{\infty}+\theta\right) \cos (\phi)-T_{\mathrm{m}}^{1 / 2} \frac{v}{\chi} \cos (\Omega t)$.

The associated boundary conditions at $z \rightarrow \infty$ are

$$
\begin{aligned}
& u \rightarrow 0, \\
& v \rightarrow 0,
\end{aligned}
$$

at $z=h$ are

$\frac{\partial u_{+}}{\partial z}=\Lambda \sqrt{\frac{H}{\Pi(1)}}\left(u_{+}-u_{-}\right)$

$\frac{\partial v_{+}}{\partial z}=\Lambda \sqrt{\frac{H}{\Pi(1)}}\left(v_{+}-v_{-}\right)$

$\bar{p}_{+}=\bar{p}_{-}$.

\subsubsection{Induced flow in the mushy layer}

We have noted that Eqs. (3.13a), (3.13b) and $(3.13 \mathrm{c})$ are already the solutions describing the velocity field in the mushy layer. Namely, both the velocities in $\boldsymbol{x}$ - and $\boldsymbol{z}$-directions vanish and the velocity in $\boldsymbol{y}$-direction, with which the yawing motion is aligned, changes with the yawing angle $\phi$, and is a result of the interaction between the gravity (represented by $R_{\mathrm{m}} \theta$ ) and the buoyancy (represented by $R_{\mathrm{m}} \theta_{\infty}$ ). More precisely to say, the induced velocity in the mush is in the same direction as the gravity component along the interface, and is a periodic function changing with time with a frequency equal to the yawing angular velocity $\Omega$, and is in the same phase with the resultant external force. Please note also that the Coriolis force shows up only on the right-hand side of Eq. (3.13d), implying that the Coriolis force makes no contribution to the induced flow, while influences the buoyant component in the $z$-direction. Eq. (3.13d) also implies that, since the pressure in the $z$-direction is in balance, the resultant force in the $z$-direction is zero. This is also true in the fluid layer, as show in Eqs. (3.12a), (3.12b), (3.12c) and (3.12d).

\subsubsection{Induced flow in the fluid layer: small yawing angle case}

In the fluid layer, Eq. (3.12a) subject to the boundary conditions Eqs. (3.14a) and (3.15a) gives the long-term solution as

$u=0$,

which implies that in the fluid layer the induced velocity in the $\boldsymbol{x}$-direction (perpendicular to the yawing direction) vanishes. The long-term solution 
shall be valid after the tank experiences a few yawing cycles and is therefore representative of a general case when the tank is steadily yawing. To solve the velocity $v$ in the melt, we first assume that the yawing angle is small, or consider that the yawing amplitude $\Phi \ll 1$, yielding

$\sin (\phi) \approx \phi=\Phi \sin (\Omega t)$

As a result, Eq. (3.12b) can be rewritten as

$$
\begin{aligned}
{\left[\frac{1}{\sigma}\left(\frac{\partial}{\partial t}-\frac{\partial}{\partial z}\right)-\frac{\partial^{2}}{\partial z^{2}}\right] v=} & {\left[R_{\mathrm{t}}\left(\theta-\theta_{\infty}\right)-R_{\mathrm{c}} \Theta\right] } \\
& \times \Phi \sin (\Omega t) .
\end{aligned}
$$

This equation can be solved analytically together with the boundary conditions Eqs. (3.14b) and (3.15b) by the following procedure. We first let $v=f(z) \mathrm{e}^{\mathrm{i} \Omega t}$ and substitute it into the above equations and replace $\sin (\Omega t)$ with $\mathrm{e}^{\mathrm{i} \Omega t}$. An ordinary differential equation for $f$ can then be extracted from Eq. (3.18), which turns out to be solvable by a formal approach. The solution is

$$
\begin{aligned}
v= & \frac{\Phi R_{\mathrm{t}}\left(\theta_{\infty}-\theta_{\mathrm{i}}\right)}{\Delta_{\mathrm{t}}} \sin \left(\phi_{\mathrm{t}}+\Omega t\right) \mathrm{e}^{-(z-h)} \\
& +\frac{\Phi R_{\mathrm{c}} \theta_{\mathrm{i}}}{\Delta_{\mathrm{c}}} \sin \left(\phi_{\mathrm{c}}+\Omega t\right) \mathrm{e}^{-(z-h) / \varepsilon} \\
& -\left\{\left(1+\Lambda \sqrt{\frac{\mathscr{H}}{\Pi(1)}}\right) \frac{R_{\mathrm{t}}\left(\theta_{\infty}-\theta_{\mathrm{i}}\right)}{\Delta_{\mathrm{i}} \Delta_{\mathrm{t}}}\right. \\
& \times \sin \left[\phi_{\mathrm{t}}-\phi_{\mathrm{i}}-b(z-h)+\Omega t\right] \\
& +\left(\frac{1}{\varepsilon}+\Lambda \sqrt{\frac{\mathscr{H}}{\Pi(1)}}\right) \frac{R_{\mathrm{c}} \theta_{\mathrm{i}}}{\Delta_{\mathrm{i}} \Delta_{\mathrm{c}}} \\
& \times \sin \left[\phi_{\mathrm{c}}-\phi_{\mathrm{i}}-b(z-h)+\Omega t\right] \\
& +\Lambda \sqrt{\mathscr{H} \Pi(1) \frac{R_{\mathrm{m}}\left(\mathscr{A} \theta_{\infty}+\theta_{\mathrm{i}}\right)}{\Delta_{\mathrm{i}}}} \\
& \left.\times \sin \left[-\phi_{\mathrm{i}}-b(z-h)+\Omega t\right]\right\} \Phi \mathrm{e}^{-(z-h) / d_{v}}
\end{aligned}
$$

in which

$$
\begin{aligned}
& d_{v}=\frac{1}{((1 / 2 \sigma)+a)}, \\
& a=\left[\left(1+\sqrt{1+16 \Omega^{2} \sigma^{2}}\right) /\left(8 \sigma^{2}\right)\right]^{1 / 2}, \\
& b=\left[\left(-1+\sqrt{1+16 \Omega^{2} \sigma^{2}}\right) /\left(8 \sigma^{2}\right)\right]^{1 / 2}, \\
& \cos \phi_{\mathrm{t}}=\frac{1-(1 / \sigma)}{\Delta_{\mathrm{t}}},
\end{aligned}
$$

$\sin \phi_{\mathrm{t}}=\frac{\Omega / \sigma}{\Delta_{\mathrm{t}}}$,

$\cos \phi_{\mathrm{c}}=\frac{(1 / \varepsilon)((1 / \varepsilon)-(1 / \sigma))}{\Delta_{\mathrm{c}}}$,

$\sin \phi_{\mathrm{c}}=\frac{\Omega / \sigma}{\Delta_{\mathrm{c}}}$,

$\cos \phi_{\mathrm{i}}=\frac{1 /(2 \sigma)+a+\Lambda \sqrt{\mathscr{H} / \Pi(1)}}{\Delta_{\mathrm{i}}}$,

$\sin \phi_{\mathrm{i}}=\frac{b}{\Delta_{\mathrm{i}}}$,

$\Delta_{\mathrm{t}}=\left[(1-(1 / \sigma))^{2}+(\Omega / \sigma)^{2}\right]^{1 / 2}$,

$\Delta_{\mathrm{c}}=\left[((1 / \varepsilon)((1 / \varepsilon)-(1 / \sigma)))^{2}+(\Omega / \sigma)^{2}\right]^{1 / 2}$,

$\Delta_{\mathrm{i}}=\left[\left(\frac{1}{2 \sigma}+a+\Lambda \sqrt{\frac{\mathscr{H}}{\Pi(1)}}\right)^{2}+b^{2}\right]^{1 / 2}$.

Eq. (3.19) illustrates that the induced flow in the fluid layer varies periodically with time with a frequency equal to the yawing angular frequency $\Omega$, being similar to that of the mushy layer. The amplitude of $v$ varies along the $z$-axis, consisting of three parts in terms of different length scales. The first part is of the length scale of thermal boundary layer, varying vertically with the exponential function $\mathrm{e}^{-(z-h)}$ and will be called the thermal-layer flow. The second part is of the length scale of solute boundary layer, varying with $\mathrm{e}^{-(z-h) / \varepsilon}$ and will be called the solute-layer flow. The third part is of a length scale of yawing-modified suction layer, varying with $\mathrm{e}^{-(z-h) / d_{v}}$ and will be called the suction-layer flow because the fluid is seen moving 
vertically into the mushy layer due the upwardgrowing interface. We also note from Eq. (3.12b) that, because of the term $\partial / \partial t$, there exists a phase difference between the induced velocity and the external force. The phase difference depends primarily on $\Omega$ and $\sigma$ and is virtually $90^{\circ}$ for the ammonium chloride solution, which turns out to be the phase difference between the velocities of the fluid and the mushy layers.

From Eqs. (3.19), (3.20a), (3.20b), (3.20c), (3.20d), (3.20e), (3.20f), (3.20g), (3.20h), (3.20h), (3.20i), (3.20j), (3.20k) and (3.201) one can find that both the yawing amplitude $\Phi$ and the yawing frequency $\Omega$ are of influence to some extent on the induced velocity in the fluid layer. A larger $\Phi$ leads to a larger induced velocity and a larger yawing frequency $\Omega$ results in a smaller induced velocity. As $\Phi$ becomes larger, the component of the gravity along the melt/mush interface becomes larger, driving a more-intense flow along the interface. As $\Omega$ becomes larger, the frequency of the change of the direction of external force is accordingly higher, resulting in that the duration of the force applied in the same direction is shorter, and consequently a less-intense flow is induced.

To illustrate the induced flow in the yawing tank, we show in Figs. $3 a$ and $b$ the induced velocities in the fluid and the mushy layers, respectively. In both figures the yawing frequency $\Omega$ is fixed to be $1 \mathrm{~Hz}$ and several velocity profiles of various yawing angles are presented. The profile marked with number 1 (or the profile No. 1) corresponds to the tank in the vertical position, under which the induced velocity in the fluid layer is of the largest amplitude while that in the mushy layer is virtually zero. As yawing proceeds and the yawing angle increases (i.e. the tank moves into the left-half $\boldsymbol{y}-\boldsymbol{z}$ plane), see profiles No. 2 and 3 , the induced velocity in the fluid layer decreases in amplitude while that in the mushy layer increases. Note that at $z \approx 0.53$ (in the mush) the induced velocity remains invariably zero and beyond that point the induced flow in the mush changes the direction. For profile No. 4 , the tank moves to the position of the largest yawing angle, the induced velocity becomes zero in the fluid layer and reaches the largest value in the mushy layer. Note that the amplitude of the induced velocity in the fluid layer

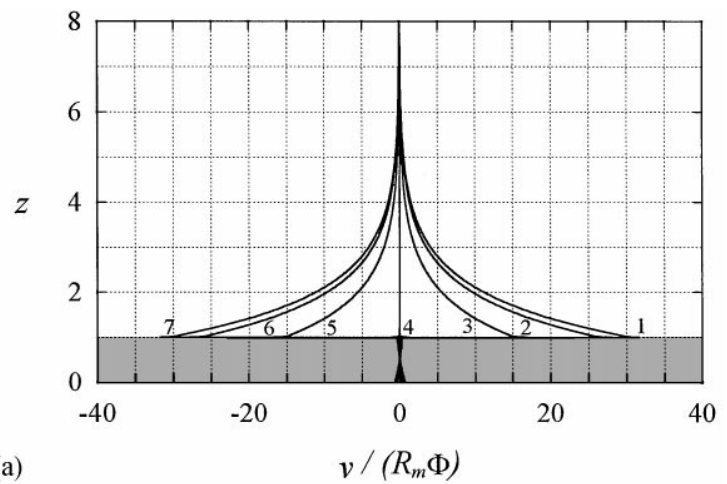

(a)

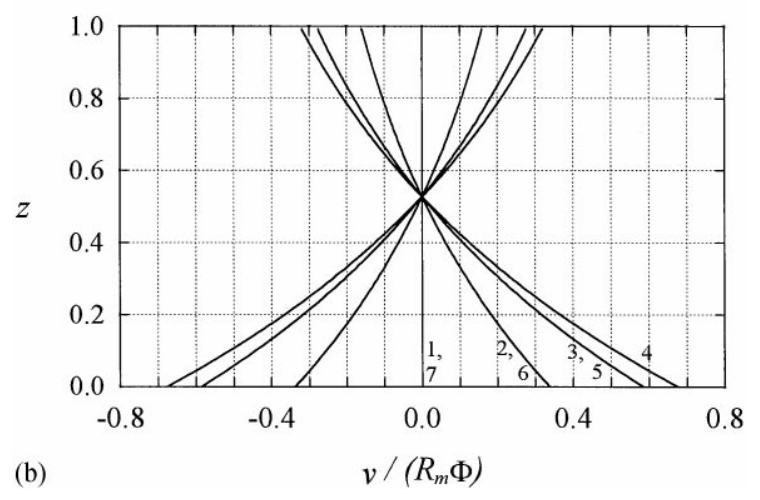

Fig. 3. The induced velocity profiles for the case of small yawing-angle with $1 \mathrm{~Hz}$ frequency $\left(\Omega=3.731 \times 10^{5}\right)$. Note that the induced velocity in the fluid layer is about 50 times larger than that in the mush. In (a) the induced velocity profiles in both the fluid and the mushy layers are shown. Profile No. 1 is for the case of $\Omega t=0$, where the tank is in the vertical position. Profile No. 2: $\Omega t=\pi / 6$; No. 3: $\Omega t=\pi / 3$; No. 4: $\Omega t=\pi / 2$ (maximum yawing-angle position); No. $5: \Omega t=2 \pi / 3$; No. $6: \Omega t=5 \pi / 6$; No. 7: $\Omega t=\pi$ (vertical position). In (b) the induced velocity profile in the mushy layer are enlarged. It is shown that the velocity at about $z=0.53$ is invariably zero, at which the induced velocity changes direction. The curves marked with numbers correspond to the counterparts of (a) while those without the marking number correspond to the cases for $\pi \leqslant \Omega t \leqslant 2 \pi$. Note that as $\Phi$ increases, the amplitude of the induced velocity also proportionally increases.

is about 50 times larger than that in the mushy layer.

As the tank moves backward to the vertical position (still in the left-half $\boldsymbol{y}-\boldsymbol{z}$ plane), the induced velocity in the fluid layer starts to increase whereas the direction of the flow is just opposite to the previous ones. On the other hand, the induced 
velocity in the mushy layer starts to decrease while the flow remains in the same direction (please see profiles No. 5, 6, and 7 of both figures). As the tank moves to the right-half $\boldsymbol{y}-\boldsymbol{z}$ plane, the induced velocity in the fluid layer changes from profile No. 7 in sequence to No. 1 when the tank experiences another yawing cycle. Meanwhile, the induced velocities in the mushy layer are of the profiles shown on the left-half plane of Fig. 3b, being symmetric with respect to those counterparts marked with numbers on the right-half plane.

The reason why the induced flow vanishes invariably at $z=0.53$ is given in the following. The curve of $\rho$ in Fig. $2 b$ indicates that $\rho(z=0.53)=$ $\rho(z=\infty)$. Since the melt at $z=\infty$ is quiescent, the buoyancy component along the interface is equal to the weight of the fluid at $z=\infty$. Therefore, the buoyancy component in the mush below $z=0.53$ is larger than the fluid's weight while is less above $z=0.53$, leading to that the fluids in the mush below and above $z=0.53$ move in different directions.

Please note that since the induced velocities in the fluid and the mushy layers are out of phase by about $90^{\circ}$, there accordingly exists a slip of the induced velocity across the melt/mush interface. This can be satisfied because of the prescription of the Beavers-Joseph condition Eq. (2.9h) or Eq. $(2.19 \mathrm{~h})$ or Eq. (3.15b). The phase of the induced velocity in the fluid layer lags by about $90^{\circ}$ behind the phase of the yawing motion, while the induced velocity in the mushy layer is of the same phase as the yawing motion. Since the induced velocity in the fluid layer changes dramatically in the vicinity above the melt/mush interface, we show in Fig. 4 a series of enlarged velocity profiles in the shallow region above the interface. In Fig. 4a the tank moves to the vertical position and the induced velocity in the fluid layer is the largest. As time goes on, the yawing angle of the tank increases while the induced velocity decreases (Figs. $4 \mathrm{~b}$ and c) and virtually vanishes (except in a small region above the interface) when the yawing angle reaches its maximum value (Fig. 4d). As the tank moves backward or the yawing angle decreases, the induced velocity increases (Figs. 4e and f) and reaches its largest amplitude when the tank returns to the vertical position (Fig. 4g). Similar results apply to Figs. $4 \mathrm{~h}-1$ in which the tank moves to the other half-plane, starting another cycle of yawing motion.

As one can see from Figs. 3 and 4, the induced velocity has a sharp gradient right above the melt/ mush interface and reduces the amplitude gradually in the $z$-direction. The induced flow stays in the same plane (i.e. the $\boldsymbol{y}-\boldsymbol{z}$ plane, see Fig. 1) as that of the yawing motion, implying that the forces in both $\boldsymbol{x}$ - and $\boldsymbol{z}$-directions do not lose their balance due to yawing. Only in the $y$-direction the balance of the external forces changes as yawing angle varies.

It is worth noting that, as can be seen from Eq. (3.19), the yawing angular frequency $\Omega$ is of significant influence on the velocity of the induced flow. We show an example in Fig. 5 where the yawing angular frequency is reduced to $0.1 \mathrm{~Hz}$ (or $\Omega=$ $3.731 \times 10^{4}$ ). All the velocity profiles in Fig. 5 are of the same yawing situation as those counterparts shown in Fig. 3. It is seen that, generally, the maximum velocity in Fig. 5 is about ten times larger than the counterpart in Fig. 3, showing that the amplitude to the maximum velocity is inversely proportional to the yawing angular frequency $\Omega$. This implies that to get an induced flow with larger velocity, the cooling tank shall not have a yawing motion with high yawing angular frequency. On the other hand, the induced flow in the mush turns out to be not influenced by the change of the yawing angular frequency, which is obviously due to the high resistance of the dendritic mush to the flow.

\subsubsection{Induced flow in the fluid layer: arbitrary yawing-angle case}

In the above section, the yawing amplitude $\Phi$ is assumed very small such that $\sin (\phi) \approx \phi=$ $\Phi \sin (\Omega t)$. This assumption will be lifted in the present section; namely, the yawing amplitude can be arbitrarily large. We employ the Taylor series expansion for $\sin (\phi)$

$$
\begin{aligned}
\sin (\phi)= & \Phi \sin (\Omega t)+\Phi^{3}\left[-\frac{1}{8} \sin (\Omega t)+\frac{1}{24} \sin (3 \Omega t)\right] \\
& +\Phi^{5}\left[\frac{1}{192} \sin (\Omega t)-\frac{1}{384} \sin (3 \Omega t)\right. \\
& \left.+\frac{1}{1920} \sin (5 \Omega t)\right]+\mathrm{O}\left(\Phi^{7}\right) .
\end{aligned}
$$




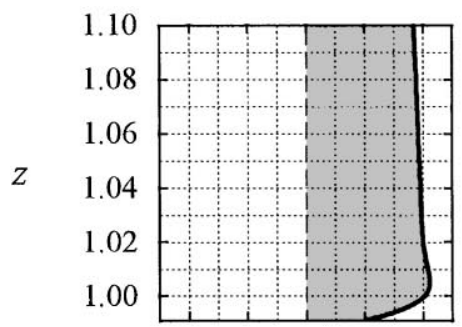

(a)

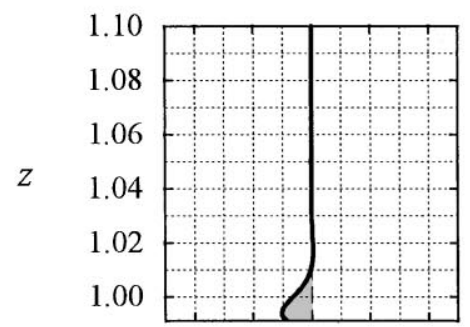

(d)

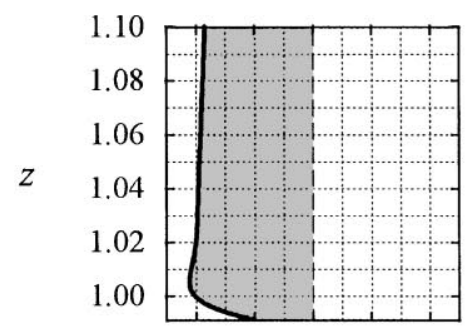

(g)

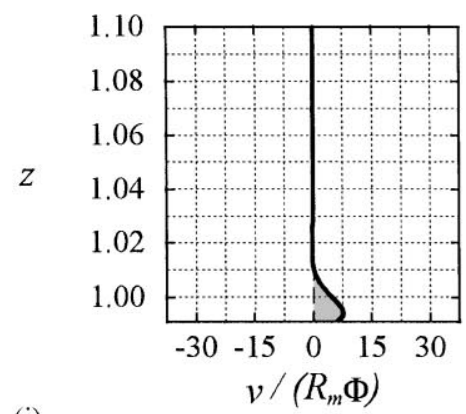

(j)

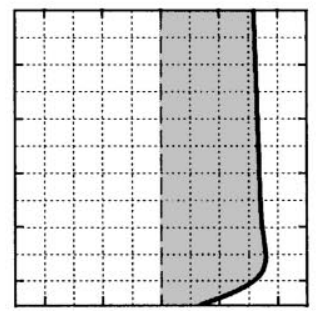

(b)

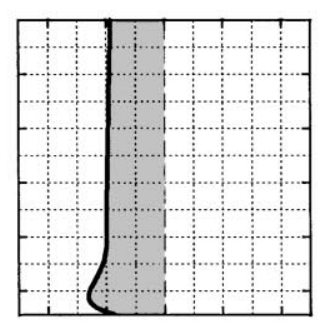

(e)

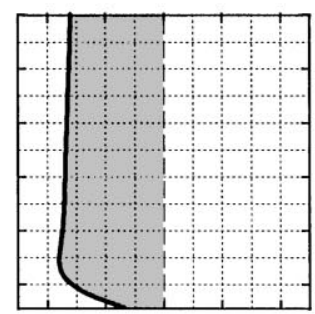

(h)

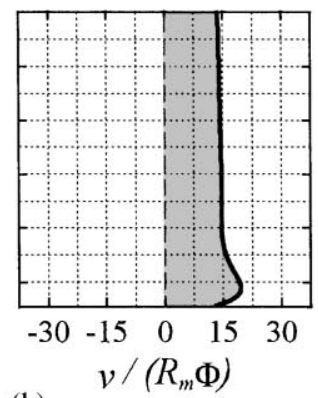

(k)

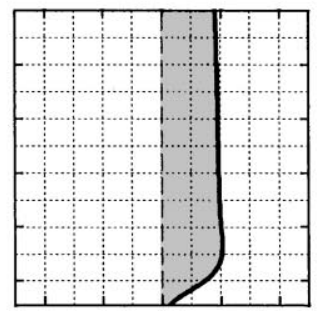

(c)

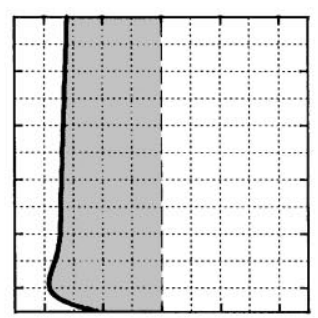

(f)

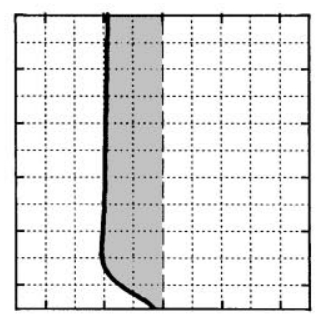

(i)

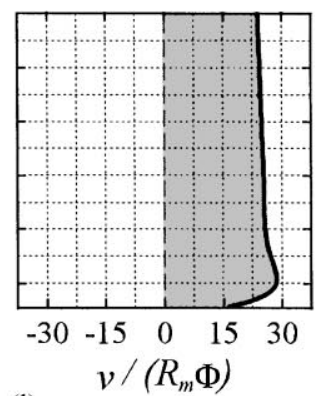

(1)

Fig. 4. The enlarged velocity profiles in the vicinity above the melt/mush interface. (a) $\Omega t=0$, when the tank is in the vertical position; (b) $\Omega t=\pi / 6$; (c) $\Omega t=\pi / 3$; (d) $\Omega t=\pi / 2$, when the tank is in the largest yawing-angle position; (e) $\Omega t=2 \pi / 3$; (f) $\Omega t=5 \pi / 6$; (g) $\Omega t=\pi$, when the tank is in the vertical position; (h) $\Omega t=7 \pi / 6$; (i) $\Omega t=4 \pi / 3$; (j) $\Omega t=3 \pi / 2$, when the tank is in the largest yawing-angle position; (k) $\Omega t=5 \pi / 3$; (l) $\Omega t=11 \pi / 6$. 

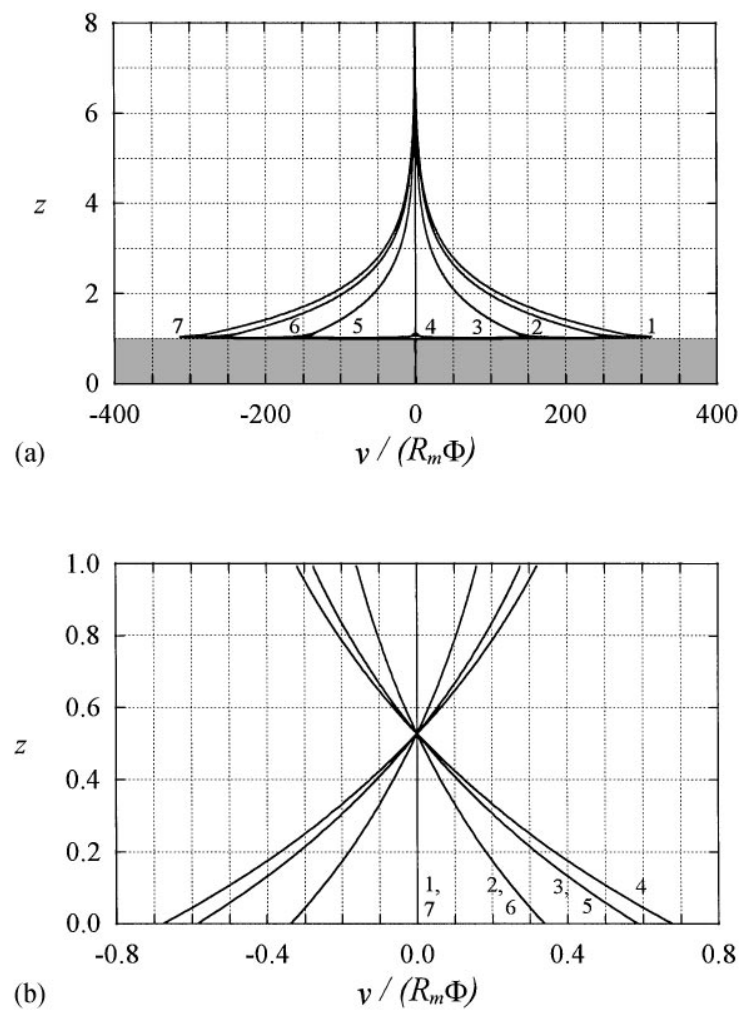

Fig. 5. Similar to Fig. 3 with a smaller frequency $0.1 \mathrm{~Hz}$ $\left(\Omega=3.731 \times 10^{4}\right)$. Note that as the yawing frequency decreases by 10 times, the induced velocity in the fluid layer increases also by 10 times while that in the mushy layer remains virtually unchanged. The cases with numbers are the same as those counterparts in Fig. 3.

By substituting above expansion into Eq. (3.18), we obtain an equation in which except for the additional higher order terms all the other terms are the same as Eq. (3.18). This equation can also be solved together with the boundary conditions (3.14b) and (3.15b) by the same approach and the solution is of the following form:

$$
\begin{gathered}
v=\left(\Phi-\frac{\Phi^{3}}{8}+\frac{\Phi^{5}}{192}+\ldots\right) V_{1} \\
+\left(\frac{\Phi^{3}}{24}-\frac{\Phi^{5}}{384}+\ldots\right) V_{3} \\
+\left(\frac{\Phi^{5}}{1920}+\ldots\right) V_{5}+\ldots,
\end{gathered}
$$

in which the $V_{j}, j=1,3,5, \ldots$, can be expressed as

$$
\begin{aligned}
V_{j}= & \frac{R_{\mathrm{t}}\left(\theta_{\infty}-\theta_{\mathrm{i}}\right)}{\Delta_{\mathrm{t} j}} \sin \left(\phi_{\mathrm{t} j}+j \Omega t\right) \mathrm{e}^{-(z-h)} \\
& +\frac{R_{\mathrm{c}} \theta_{\mathrm{i}}}{\Delta_{\mathrm{cj}}} \sin \left(\phi_{\mathrm{cj}}+j \Omega t\right) \mathrm{e}^{-(z-h) / \varepsilon} \\
& -\left\{\left(1+\Lambda \sqrt{\frac{\mathscr{H}}{\Pi(1)}}\right) \frac{R_{\mathrm{t}}\left(\theta_{\infty}-\theta_{\mathrm{i}}\right)}{\Delta_{\mathrm{i} j} \Delta_{\mathrm{t} j}}\right. \\
& \times \sin \left[\phi_{\mathrm{t} j}-\phi_{\mathrm{i} j}-b_{j}(z-h)+j \Omega t\right] \\
& +\left(\frac{1}{\varepsilon}+\Lambda \sqrt{\frac{\mathscr{H}}{\Pi(1)}}\right) \frac{R_{\mathrm{c}} \theta_{\mathrm{i}}}{\Delta_{\mathrm{i} j} \Delta_{\mathrm{c} j}} \\
& \times \sin \left[\phi_{\mathrm{c} j}-\phi_{\mathrm{i} j}-b_{j}(z-h)+j \Omega t\right] \\
& +\Lambda \sqrt{\mathscr{H} \Pi(1) \frac{R_{\mathrm{m}}\left(\mathscr{A} \theta_{\infty}+\theta_{\mathrm{i}}\right)}{\Delta_{\mathrm{i} j}}} \\
& \left.\times \sin \left[-\phi_{\mathrm{i} j}-b_{j}(z-h)+j \Omega t\right]\right\} \mathrm{e}^{-(z-h) / d_{j}} .
\end{aligned}
$$

In the above equation,

$$
d_{j}=\frac{1}{\left((1 / 2 \sigma)+a_{j}\right)},
$$

$a_{j}=\left[\left(1+\sqrt{1+16 j^{2} \Omega^{2} \sigma^{2}}\right) /\left(8 \sigma^{2}\right)\right]^{1 / 2}$,

$b_{j}=\left[\left(-1+\sqrt{1+16 j^{2} \Omega^{2} \sigma^{2}}\right) /\left(8 \sigma^{2}\right)\right]^{1 / 2}$,

$\cos \phi_{\mathrm{t} j}=\frac{1-(1 / \sigma)}{\Delta_{\mathrm{t} j}}$,

$\sin \phi_{\mathrm{t} j}=\frac{j \Omega / \sigma}{\Delta_{\mathrm{t} j}}$,

$\cos \phi_{\mathrm{c} j}=\frac{(1 / \varepsilon)((1 / \varepsilon)-(1 / \sigma))}{\Delta_{\mathrm{c} j}}$,

$\sin \phi_{\mathrm{c} j}=\frac{j \Omega / \sigma}{\Delta_{\mathrm{c} j}}$

$\cos \phi_{\mathrm{i} j}=\frac{1 /(2 \sigma)+a_{j}+\Lambda \sqrt{H / \Pi(1)}}{\Delta_{\mathrm{i} j}}$, 
$\sin \phi_{\mathrm{i} j}=\frac{b_{j}}{\Delta_{\mathrm{i} j}}$

$\Delta_{\mathrm{t} j}=\left[(1-(1 / \sigma))^{2}+(j \Omega / \sigma)^{2}\right]^{1 / 2}$,

$\Delta_{\mathrm{c} j}=\left[((1 / \varepsilon)((1 / \varepsilon)-(1 / \sigma)))^{2}+(j \Omega / \sigma)^{2}\right]^{1 / 2}$,

$\Delta_{\mathrm{i} j}=\left[\left(\frac{1}{2 \sigma}+a_{j}+\Lambda \sqrt{\frac{\mathscr{H}}{\Pi(1)}}\right)^{2}+b_{j}^{2}\right]^{1 / 2}$.

Note that $V_{j}$ is also a time-periodical function, changing with the angular frequency $j \Omega$. The firstorder solution $\Phi V_{1}$ of Eq. (3.22) is exactly the same as the solution for small yawing-angle case, Eq. (3.19). It is seen that in the case of finite yawing angle $\phi$, the induced velocity consists of infinite number of oscillatory modes with different frequencies, such as $3 \Omega, 5 \Omega$, and so on. While for the case of infinitesimal yawing-angle, the induced velocity contains only one mode with frequency $\Omega$. We nevertheless find that increasing yawing-angle only changes the induced velocity by a little amount, as shown in Fig. 6. In this case, the yawing angle increases to $60^{\circ}$, equivalent to $\Phi=1.05$, the induced velocity actually decreases by less than $1 \%$. This can be also seen from Eq. (3.22) that the higher-order terms of $\Phi^{n}$ are of small contribution to the value of $v$. Results shown in Fig. 6 imply that there may be no need to consider the case of finite yawing-angle because the solution of small yawing-angle is of much simplified form and can already reveal the major features of the induced flow.

\section{Conclusions}

The present analysis shows that the yawing motion is another scheme, with which a parallel shear flow in the cooling tank can be successfully induced. This scheme is to some extent similar to that proposed by Sample and Hellawell [9] in which the tank was forced to spin and/or precess with respect to an inclined axis. As inferred by Sample and Hellawell, the suppression of the plume convection in their system is a result of the occurrence of the shear flow induced by the inclined-spin and/or inclined-precession motion of the tank. This
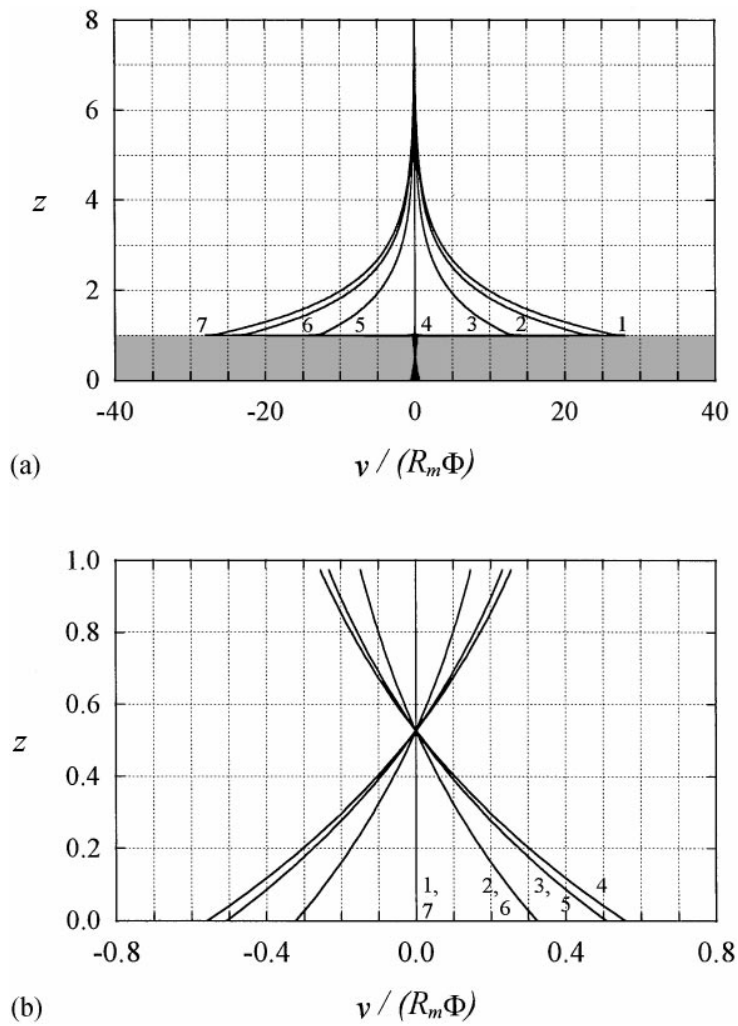

Fig. 6. The induced velocity profiles for the case of large yawing amplitude $\left(\Phi=1.05\right.$ or $\left.60^{\circ}\right)$ with $1 \mathrm{~Hz}$ frequency $\left(\Omega=3.731 \times 10^{5}\right)$. Please note that the amplitude of the induced velocity obtained by the arbitrary-large $\Phi$ model is generally smaller than that obtained by the small- $\Phi$ model. For the arbitrary-large $\Phi$ model, although some small correction factors are included, as shown in Eq. (3.22), the amplitude of the induced velocity is also virtually proportional to the yawing amplitude.

shall apply also to the present system: the tank shakes with a yawing motion in the vertical plane.

We have shown by an analytical approach that there exists an induced parallel shear flow in both the fluid and the mushy layers when the tank is yawing in a vertical plane. The induced flow in the fluid layer is of much larger velocity than that in the mushy layer. The induced flow in the mushy layer is of the same phase as the yawing motion while is about $90^{\circ}$ out of phase with the induced flow in the fluid layer. There accordingly exist a slip of the horizontal velocity across the melt/mush interface, resulting in the formation of a sharp velocity gradient above the interface. It is this sharp velocity 
gradient which can effectively suppress the occurrence of the boundary-layer mode convection $[2,4]$ or the salt-finger convection [1]. As a result, the formation of the plume convection, which is related directly to the formation of freckles in castings, is also very likely to be suppressed due to the absence of the boundary-layer mode convection [6-8].

We have also pointed out that the amplitude of the induced velocity in the melt is virtually inversely proportional to the yawing frequency while that in the mush is completely independent of the yawing frequency because there is no time-dependent term in the Darcy equation of the mush. On the other hand, however, the amplitude of the induced velocity in both layers is proportional to the yawing amplitude. Knowing these characteristics of the induced flow, an efficient way based on the yawing motion of the tank can be set up and then applied in the manufacturing process of directional solidification.

\section{Acknowledgements}

The financial support for this work from National Science Council through Grant No. 2212-E-002-
018 is gratefully acknowledged. The post-doctoral fellowship for C.A.C. from the same grant is also appreciated.

\section{References}

[1] C.F. Chen, F. Chen, J. Fluid Mech. 227 (1991) 567.

[2] M.G. Worster, J. Fluid Mech. 237 (1992) 649.

[3] S. Tait, C. Jaupart, J. Geophys. Res. 97 (B5) (1992) 6735.

[4] F. Chen, J.W. Lu, T.L. Yang, J. Fluid Mech. 276 (1994) 163.

[5] F. Chen, J. Crystal Growth 179 (1997) 277.

[6] G. Amgerg, G.M. Homsy, J. Fluid Mech. 252 (1993) 79.

[7] D.M. Anderson, M.G. Worster, J. Fluid. Mech. 302 (1995) 307.

[8] C.A. Chung, F. Chen, J. Fluid Mech. (1999), in press.

[9] A.K. Sample, A. Hellawell, Metall. Trans. A 15 (1984) 2163.

[10] D.G. Neilson, F.P. Incropera, Int. J. Heat Mass Transfer 36 (1992) 489.

[11] J.W. Lu, F. Chen, Int. J. Heat Mass Transfer 40 (1997) 237.

[12] H.E. Huppert, M.A. Hallworth, J. Crystal Growth 130 (1993) 495.

[13] M.G. Worster, J. Fluid Mech. 167 (1986) 481.

[14] G.S. Beavers, D.D. Joseph, J. Fluid Mech. 30 (1967) 199. 